\title{
Cáncer de próstata metastásico hormono-sensible: ¿Cómo deberíamos tratar a nuestros pacientes?
}

\section{Hormone-sensitive metastatic prostate cancer: How should we treat our patients?}

" Ray Manneh Kopp, MD MSc., 2 *

'Sociedad de Oncología y Hematología del Cesar, Valledupar - Colombia. ${ }^{2}$ Grupo de Oncología Genitourinaria de Colombia (GOGUC).

Doi: https://doi.org/10.51643/22562915.74

ए I cáncer de próstata es la neoplasia maligna más frecuente en varones; en nuestro país se estima que hasta un $30 \%$ de los pacientes padecen una enfermedad en situación avanzada e incurable.

Los pacientes con enfermedad metastásica son un grupo heterogéneo, su pronóstico varía de manera muy marcada en función de la forma de debut, los tratamientos iniciales previos y el volumen de la enfermedad al diagnóstico'.

Basándonos en esto, podemos dividir a los pacientes en cuatro grandes grupos, unas medianas de supervivencia peores en función de la forma de debut y el volumen de la enfermedad. Así, los pacientes con bajo volumen de la enfermedad y que presentan una recidiva metastásica hormono-sensible, tendrán unas medianas de supervivencia que rondan los 8 años; en cambio, los pacientes con enfermedad metastásica de novo y un alto volumen, sólo alcanzan unas medianas de supervivencia aproximadas de tres años.

Entre estos dos extremos quedan los pacientes que presentan recidiva metastásica de alto volumen, y aquellos que debutan con metástasis, pero con bajo volumen: se estima que la supervivencia de estos dos grupos de pacientes, rondaría los 4,5 años ${ }^{2}$.

El cáncer de próstata metastásico hormono-sensible (CPMHS), es una situación clínica de la enfermedad que ha recobrado mucho interés en los últimos cinco años. Desde los resultados del estudio CHAARTED $^{3}$, sumados a los del STAMPEDE ${ }^{4}$, pudimos evidenciar, que el docetaxel, añadido a la terapia de deprivación androgénica, aporta un clarísimo beneficio en términos de supervivencia global (entre 15 y 17 meses).

Dicho beneficio es más marcado en pacientes de alto volumen (cuatro o más lesiones metastásicas óseas y al menos una fuera del esqueleto axial o metástasis viscerales). Para los autores del CHAARTED, el docetaxel debería limitarse a pacientes con enfermedad de alto volumen, sin embargo, el grupo británico defiende su uso en el total de la población, argumentando que todos los subgrupos se benefician y que no se pueden excluir los pacientes de bajo volumen, basándonos en un análisis no pre-planeado.

En el 2017, se comunicaron los resultados del estudio LATITUDE, un estudio fase III que randomizaba a los pacientes con CPMHS de "alto riesgo" (dos de tres de los siguientes criterios: Gleason 8 o más, tres o más metástasis óseas o metástasis viscerales), a recibir Acetato de Abiraterona + prednisona vs terapia de deprivación androgénica (TDA). Los resultados, nuevamente sorprendentes, mostraron un claro beneficio de añadir acetato de abiraterona en este escenario, mejorando la supervivencia en unos 17 meses y disminuyendo el riesgo de muerte en un $44 \% 5$. El estudioSTAMPEDE en sus brazos que comparaban acetato de abiraterona/prednisolona + TDA vs TDA, confirmaba estos resultados y al incluir pacientes con enfermedad localmente avanzada y de bajo volumen, sugería un beneficio en una población más amplia6 .

Siguiendo la línea del tiempo, cambiando la 
estrategia, el grupo británico del estudio STAMPEDE comparó el tratamiento estándar TDA +/- docetaxel vs radioterapia sobre el tumor primario + TDA +/- docetaxel. El objetivo primario era la supervivencia global. El estudio no mostró diferencias significativas en la totalidad de la población, pero al analizar la población de bajo volumen, se identifica un claro beneficio, con una reducción del riesgo de muerte de un $32 \%$. Aunque no era un análisis pre-planeado, la población estaba estratificada y los grupos estaban bien balanceados, con lo cual, los autores concluyen que la radioterapia en la enfermedad de bajo volumen, debería considerarse, dado que, además no hay diferencias significativas en términos de toxicidad grado 3-57

En el congreso de ASCO GU del 2019 se comunicaron los resultados del estudio ARCHES, incluyendo una población con alto y bajo volumen, comparando Enzalutamida + TDA vs TDA + placebo y permitiendo una pequeña población de pacientes que habían recibido docetaxel previamente. El estudio mostró mejoría en la supervivencia libre de progresión radiológica (su objetivo primario), con una reducción del riesgo de progresión o muerte del $61 \%$.

Unos meses más tarde, en la plenaria de ASCO 2019, se comunicaron los resultados del estudio ENZAMET, el cual incluía pacientes con bajo y alto volumen, permitía el uso concurrente de docetaxel (que se planeó hacer en un $44 \%$ de los pacientes) y su objetivo principal era supervivencia global. Los resultados, nuevamente positivos, mostraron una disminución del riesgo de muerte de un 33 \% . En esa misma reunión, también se comunicaron los resultados del estudio TITAN, otro estudio fase III,

que probaba en la misma población de pacientes, Apalutamida + TDA vs TDA, también permitía el uso de docetaxel previo (no concurrente), al igual que ARCHES y ENZAMET, abarcaba todo el espectro de la enfermedad hormono-sensible, con pacientes de bajo y alto volumen. El estudio mostró un beneficio en términos de supervivencia libre de progresión radiológica (disminución del riesgo de progresión o muerte del 52\%) y de supervivencia global, logrando disminuir el riesgo de muerte en un $33 \%{ }^{10}$.

Los datos de estos tres últimos estudios, aún son inmaduros y aunque demuestran un claro beneficio en sus objetivos, falta seguimiento para intentar determinar diferencias y subpoblaciones de interés.

Al parecer, los análisis por subgrupo (aunque aún falta tiempo de seguimiento), no terminan de demostrar el beneficio de enzalutamida o apalutamida en pacientes que reciben previamente docetaxel en la fase de hormono-sensibilidad.

Lo que hasta ahora demuestran TITAN y ENZAMET, es que el beneficio de los nuevos fármacos inhibidores del eje androgénico es independiente del volumen de la enfermedad, que tienen un perfil de toxicidad manejable y que posicionan a enzalutamida y apalutamida como alternativas válidas a la hora de escoger los tratamientos en esta población.

A modo de resumen, para pacientes con alto volumen, aptos para recibir quimioterapia, tenemos como opciones: docetaxel, abiraterona, apalutamida, enzalutamida.

En pacientes de alto volumen, no aptos para quimioterapia: abiraterona, apalutamida, enzalutamida. En pacientes de bajo volumen: abiraterona, apalutamida, enzalutamida, docetaxel (sólo en los aptos a quimioterapia), considerando siempre la posibilidad de la radioterapia sobre el tumor primario.

Tal vez lo más importante que hemos aprendido en los últimos años, es que la TDA no es suficiente y que es nuestro deber ofrecer alguno de los fármacos o estrategias comentadas anteriormente, al menos, a la gran mayoría de nuestros pacientes. 


\section{Referencias}

1. Francini E, Gray K, Xie W. Time of metastatic disease presentation and volume of disease are prognostic for metastatic hormone sensitive prostate cancer (mHSPC). Prostate Sep;78 (12):889-895. https://doi.org/10.1002/ pros. 23645 .

2. Gravis G, Boher J-M, Chen Y-H. Burden of metastatic castrate naive prostate cancer patients, to identify men more likely to benefit from early docetaxel: further analyses of CHAARTED and GETUG_AFU15 studies. Eur Urol Jun;73 (6):847-855. https://dx.doi. org/10.1016\%2Fj.eururo.2018.02.001.

3. Sweeney C, Chen Y-H, Carducci M. Chemohormonal therapy in metastatic hormone-sensitive prostate cancer. N Engl J Med 2015; 373:737-746. https://doi.org/10.1200/ jco.2017.75.3657.

4. James N, Sydes M, Clarke N, Mason M, Dearnaley $D$, Spears $M$, et al. Addition of docetaxel, zoledronic acid, or both to first-line long-term hormone therapy in prostate cancer (STAMPEDE): survival results from an adaptive, multiarm, multistage, platform randomized controlled trial. Lancet.2016 Mar 19;387 (10024): 1163-77. https://doi. org/10.1016/s0140-6736(15)01037-5.

5. Fizazi K, Tran N, Fein L, Matsubara N, Rodriguez-Antolin A, Alekseev B, et al. Abiraterone plus prednisone in metastatic castration-sensitive prostate cancer. N Engl J Med 2017. Jul 27;377(4):352-360. https://doi. org/10.1056/nejmoa1704174.
6. James N, de Bono J, Spears M. Abiraterone for prostate cancer not previously treated with hormone therapy. N Engl J Med. 2017 Jul 27;377(4):338-35. https://goi.org/10.1056/ NEJMoa1702900.

7. Parker C, James N, Brawley C. Clarke N, Hoyle A, Ali A, et al. Radiotherapy to the primary tumour for newly diagnosed, metastatic prostate cancer (STAMPEDE): a randomized controlled phase 3 trial. Lancet 2018 Dec 1;392 (10162):2353-2366. https:// doi.org/10.1016/s0140-6736(18)32486-3.

8. Amstrong A, Szmulewitz R, Petrylak D, Holzbeierlein J, Villers A, Azad A, et al. ARCHES: a randomized, phase III study of androgen deprivation therapy with enzalutamide of placebo in men with metastatic hormonse-sensitive prostate cancer. J Cllin Oncol. 2019 Nov 10;37(32): 2974-2986. https://doi. org/10.1200/jco.19.00799.

9. Davis I, Martin A, Stockler M, Begbie S, Chi KN, Chowdhury S, et al. Enzalutamide with standard first-line therapy in metastatic prostate cancer. N Engl J Med. 2019 Jul 11;381(2);121-131. https://doi.org/10.1056/nejmoa1903835.

10. Chi K, Agarwal N, Bjartell A, Chung BH, Pereira de Santana Gomes AJ, Given R, et al. Apalutamide for metastatic, castration-sensitive prostate cancer. N Engl J Med 2019 Jul 4;381(1):13-24. https://doi.org/10.1056/nejmoa1903307 